\title{
Evaluating $L$-functions with few known coefficients
}

\author{
David W. Farmer and Nathan C. Ryan
}

\begin{abstract}
We address the problem of evaluating an $L$-function when only a small number of its Dirichlet coefficients are known. We use the approximate functional equation in a new way and find that it is possible to evaluate the $L$-function more precisely than one would expect from the standard approach. The method, however, requires considerably more computational effort to achieve a given accuracy than would be needed if more Dirichlet coefficients were available.
\end{abstract}

\section{Introduction}

$L$-functions are central to much of contemporary number theory. Two celebrated conjectures, the Riemann hypothesis and the Birch and Swinnerton-Dyer conjecture, are about values of $L$-functions and were discovered as a result of the explicit computation of the Riemann zeta function and the Hasse-Weil $L$-function associated to an elliptic curve, respectively. These $L$-functions are, respectively, of degree one and degree two and it is interesting to verify analogous conjectures about special values and zeros for higher degree $L$-functions. Conjectures such as Böcherer's conjecture $[\mathbf{5}, \mathbf{1 7}]$ and the Bloch-Kato conjecture [3] are about the central values of degree four and degree three $L$-functions, and the Grand Riemann hypothesis asserts that all nontrivial zeros of an $L$-function, of any degree, lie along the critical line.

In addition to these conjectures, there are a number of other conjectures for the statistical behavior of $L$-functions, arising from the interplay between random matrix theory and number theoretic heuristics $[\mathbf{6}, \mathbf{7}, \mathbf{1 0}]$. One of the main reasons those conjectures are believable is that large-scale calculations of the value distribution and the zeros of $L$-functions yield data that support those conjectures.

The $L$-functions we consider here are associated to Siegel modular forms. Our examples will use the first non-lift Siegel modular form on $\operatorname{Sp}(4, \mathbb{Z})$. The form has weight 20 and is usually denoted $\Upsilon_{20}$. Background information beyond what we mention about Siegel modular forms can be found in $[\mathbf{2}, \mathbf{1 1}, \mathbf{1 8}]$. For this paper the relevant information is that a Siegel modular form is acted on by Hecke operators $T(n)$, which have eigenvalues $\lambda(n)$. It is the eigenvalues $\lambda(p)$ and $\lambda\left(p^{2}\right)$, for $p$ prime, which are used to define the $L$-functions associated to the modular form. For $\Upsilon_{20}$ the eigenvalues $\lambda(p)$ have been computed for $p \leqslant 997$, and the eigenvalues $\lambda\left(p^{2}\right)$ for $p \leqslant 79$ [12]. These data are available at [19].

There is an $L$-function $L(s, F, \rho)$ of degree $n$ for each $n$-dimensional representation $\rho$ of the dual group of PGSp(4), namely $\operatorname{Sp}(4, \mathbb{C})$. Associated to a Siegel modular form $F$ is a sequence of $L$-functions, of degrees $4,5,10,14,16$, etc. The degree 4,5 , and $10 L$-functions are called, respectively, the spinor, standard, and adjoint, and are denoted $L(s, F, \operatorname{spin}), L(s, F$, stan), and $L(s, F$, adj). Proposition 2.1, taken from [9], summarizes the properties of those $L$-functions for a weight $k$ Siegel modular form on $\operatorname{Sp}(4, \mathbb{Z})$.

The degree 4 and $5 L$-functions were shown by Andrianov [1] and Böcherer [4] to have an analytic continuation and satisfy a functional equation. The degree $10 \mathrm{~L}$-function was only recently shown by Pitale, Saha, and Schmidt [14] to have an analytic continuation and satisfy

Received 31 December 2012; revised 9 October 2013.

2010 Mathematics Subject Classification 11F66, 11 Y35 (primary). 
a functional equation. Those properties for the $L$-functions of degree 14 and above are still conjectural.

\subsection{Evaluating $L$-functions}

We are concerned with numerically evaluating $L$-functions. The standard tool, which is used in available open-source computational packages $[\mathbf{8}, \mathbf{1 5}]$, is the approximate functional equation. See Proposition 3.1 for the precise formulation.

There are two main difficulties in evaluating high-degree $L$-functions. The first is that if the $L$-function $L(s)$ has degree $d$, evaluating $L\left(\frac{1}{2}+i t\right)$ using the approximate functional equation requires $\gg(1+|t|)^{d / 2}$ Dirichlet series coefficients. Here the implied constant depends on the $L$-function and the desired precision in the answer. For example, estimating the implied constant from the calculations in $\S 4$, to find the first one million zeros of $L\left(s, \Upsilon_{20}\right.$, adj $)$, the degree $10 \mathrm{~L}$-function associated to $\Upsilon_{20}$, would require using the approximate functional equation with around $10^{30}$ Dirichlet series coefficients. Note that one million zeros is not even a large sample; for example it is probably not sufficient for testing various conjectures about the lower-order terms in the distribution of spacings between zeros.

The second difficulty is that current methods are incapable of producing a large number of Dirichlet coefficients of the standard and adjoint $L$-functions of a Siegel modular form. The Fourier coefficients indexed by quadratic forms with discriminant up to 3000000 have been computed for $\Upsilon_{20}$ [12]. These Fourier coefficients are used to compute the Hecke eigenvalues. Examination of formulas on $[\mathbf{1 8}$, p. 387] shows that to find the eigenvalue $\lambda(n)$ of $T(n)$, for $n=p^{2}$, requires the Fourier coefficients indexed by quadratic forms of discriminant up to $n^{2}=p^{4}$.

It gets worse. By (2.2) and (2.4), the $p$ th Dirichlet coefficient of the standard or adjoint $L$-function requires both $\lambda(p)$ and $\lambda\left(p^{2}\right)$. Thus, to determine the first $n$ Dirichlet coefficients of those $L$-functions requires Fourier coefficients of the Siegel modular form of index up to approximately $n^{4}$. The extensive calculations in [12] are not even sufficient to determine the 83rd Dirichlet coefficient of the standard or adjoint $L$-functions of $\Upsilon_{20}$.

Of course, one could determine more Dirichlet coefficients by first finding more Fourier coefficients of the cusp form. But the $n^{4}$ relationship makes this quite expensive, so with current methods it is not feasible to determine many more Dirichlet coefficients than currently known. It is possible that new methods will be developed to determine the Hecke eigenvalues without extensive computation. The real problem will still remain: how to compute high-degree $L$-functions without requiring an enormous number of Dirichlet coefficients.

That brings us to the theme of this paper: how accurately can one compute an $L$-function given a limited number of Dirichlet coefficients? As the above discussion indicates, this is a practical problem and there are many $L$-functions for which it is not currently possible to determine a reasonably large number of Dirichlet coefficients.

As we describe in this paper, even without knowing many Dirichlet coefficients, we were able to evaluate the $L$-functions to surprisingly high accuracy (surprising to us, anyway). In fact, we were not able to establish that there is an absolute limit to the accuracy one can obtain from only a limited number of coefficients. However, our method is computationally expensive, much more expensive than evaluating the $L$-function in a straightforward way if more coefficients were available. If one could find an efficient way to determine the unknown parameters in our method, that could make it possible to quickly evaluate high-degree $L$-functions. See $\S 6$ for a discussion.

In the next section we describe the $L$-functions we consider here, and in $\S 3$ we recall the approximate functional equation and how it is used to compute an $L$-function. In $\S 4$ we state the underlying problem and then describe our experiments evaluating $L\left(s, \Upsilon_{20}\right.$, stan) and $L\left(s, \Upsilon_{20}\right.$, adj). In $\S 5$ we describe a second method that performs a little better than the 
method in $\S 4$ and show how this second method can be used to approximate unknown Dirichlet series coefficients.

\section{The L-functions}

The $L$-functions associated to a Siegel modular form are most conveniently described as Euler products. The local factors of the Euler product can be expressed in terms of the Hecke eigenvalues $\lambda(p)$ and $\lambda\left(p^{2}\right)$, but it is more convenient to express them in terms of the Satake parameters, $\alpha_{0, p}, \alpha_{1, p}$, and $\alpha_{2, p}$, given by

$$
\begin{aligned}
p^{2 k-3} & =\alpha_{0}^{2} \alpha_{1} \alpha_{2}, \\
A & =\alpha_{0}^{2} \alpha_{1}^{2} \alpha_{2}+\alpha_{0}^{2} \alpha_{1} \alpha_{2}^{2}+\alpha_{0}^{2} \alpha_{1}+\alpha_{0}^{2} \alpha_{2}, \\
B & =\alpha_{0}^{2} \alpha_{1}^{2} \alpha_{2}^{2}+\alpha_{0}^{2} \alpha_{1}^{2}+\alpha_{0}^{2} \alpha_{2}^{2}+\alpha_{0}^{2},
\end{aligned}
$$

where

$$
\begin{aligned}
& \lambda(p)^{2}=4 p^{2 k-3}+2 A+B \\
& \lambda\left(p^{2}\right)=(2-1 / p) p^{2 k-3}+A+B
\end{aligned}
$$

We suppress the $p$ on the Satake parameters when clear from context. See [16] for a discussion of how to solve this polynomial system of three equations for the three unknowns $\alpha_{0, p}, \alpha_{1, p}, \alpha_{2, p}$ using Gröbner bases.

We rescale the Satake parameters to have the so-called 'analytic' normalization $\left|\alpha_{j}\right|=1$, $\alpha_{0}^{2} \alpha_{1} \alpha_{2}=1$, which is possible if we assume the Ramanujan bound on the Hecke eigenvalues. This corresponds to a simple change of variables in the $L$-functions, so that all our $L$-functions satisfy a functional equation in the standard form $s \leftrightarrow 1-s$.

As an error check for the reader who may wish to extend our calculations, for $\Upsilon_{20}$ we have $\lambda(2)=-840960, \lambda(4)=248256200704$, and the Satake parameters at 2 are approximately

$$
\left\{\alpha_{0}, \alpha_{1}, \alpha_{2}\right\}=\{-0.901413+0.43296 i, 0.630904-0.775861 i,-0.211226+0.977437 i\} .
$$

Here and throughout this paper, decimal values are truncations of the true values.

Proposition 2.1. Suppose $F \in M_{k}(\operatorname{Sp}(4, \mathbb{Z}))$ is a Hecke eigenform. Let $\alpha_{0, p}, \alpha_{1, p}, \alpha_{2, p}$ be the Satake parameters of $F$ for the prime $p$, where we suppress the dependence on $p$ in the formulas below. For $\rho \in\{\operatorname{spin}, \operatorname{stan}, \operatorname{adj}\}$ we have the $L$-functions $L(s, F, \rho):=$ $\prod_{p \text { prime }} Q_{p}\left(p^{-s}, F, \rho\right)^{-1}$ where

$$
\begin{aligned}
Q_{p}(X, F, \text { spin }):= & \left(1-\alpha_{0} X\right)\left(1-\alpha_{0} \alpha_{1} X\right)\left(1-\alpha_{0} \alpha_{2} X\right)\left(1-\alpha_{0} \alpha_{1} \alpha_{2} X\right), \\
Q_{p}(X, F, \operatorname{stan}):= & (1-X)\left(1-\alpha_{1} X\right)\left(1-\alpha_{1}^{-1} X\right)\left(1-\alpha_{2} X\right)\left(1-\alpha_{2}^{-1} X\right), \\
Q_{p}(X, F, \text { adj }):= & (1-X)^{2}\left(1-\alpha_{1} X\right)\left(1-\alpha_{1}^{-1} X\right)\left(1-\alpha_{2} X\right)\left(1-\alpha_{2}^{-1} X\right) \\
& \times\left(1-\alpha_{1} \alpha_{2} X\right)\left(1-\alpha_{1}^{-1} \alpha_{2} X\right)\left(1-\alpha_{1} \alpha_{2}^{-1} X\right)\left(1-\alpha_{1}^{-1} \alpha_{2}^{-1} X\right),
\end{aligned}
$$

give the $L$-series of, respectively, the spin, standard, and adjoint $L$-functions. These $L$-functions satisfy the functional equations

$$
\begin{aligned}
\Lambda(s, F, \text { spin }) & :=\Gamma_{\mathbb{C}}\left(s+\frac{1}{2}\right) \Gamma_{\mathbb{C}}\left(s+k-\frac{3}{2}\right) L(s, F, \text { spin }) \\
& =(-1)^{k} \Lambda(1-s, F, \operatorname{spin}) \\
\Lambda(s, F, \operatorname{stan}) & :=\Gamma_{\mathbb{R}}(s) \Gamma_{\mathbb{C}}(s+k-2) \Gamma_{\mathbb{C}}(s+k-1) L(s, F, \text { stan }) \\
& =\Lambda(1-s, F, \operatorname{stan})
\end{aligned}
$$




$$
\begin{aligned}
\Lambda(s, F, \operatorname{adj}):= & \Gamma_{\mathbb{R}}(s+1)^{2} \Gamma_{\mathbb{C}}(s+1) \\
& \times \Gamma_{\mathbb{C}}(s+k-2) \Gamma_{\mathbb{C}}(s+k-1) \Gamma_{\mathbb{C}}(s+2 k-3) L(s, F, \text { adj }) \\
= & \Lambda(1-s, F, \operatorname{adj}) .
\end{aligned}
$$

In (2.5), we use the normalized $\Gamma$-functions

$$
\Gamma_{\mathbb{R}}(s):=\pi^{-s / 2} \Gamma(s) \quad \text { and } \quad \Gamma_{\mathbb{C}}(s):=2(2 \pi)^{-s} \Gamma(s) .
$$

The degree of an $L$-function is $r+2 c$ where $r$ and $c$ are the number of $\Gamma_{\mathbb{R}}$ and $\Gamma_{\mathbb{C}}$ factors in the functional equation, respectively. The spin, standard, and adjoint $L$-functions described above are of degree 4,5 , and 10 . The Ramanujan bound for a degree $d L$-function with Dirichlet series $\sum_{n \geqslant 1} b_{n} n^{-s}$ is given by

$$
\left|b_{p^{j}}\right| \leqslant\left(\begin{array}{c}
d+j-1 \\
j
\end{array}\right)
$$

Note that this is equivalent to the assertion that the Satake parameters satisfy $\left|\alpha_{j, p}\right| \leqslant 1$.

\section{The approximate functional equation}

In this section we describe the approximate functional equation, which is the primary tool used to evaluate $L$-functions. The approximate functional equation involves a test function which can be chosen with some freedom. This will play a key role in our calculations.

\subsection{Smoothed approximate functional equations}

The material in this section is taken from $[\mathbf{1 5}, \S 3.2]$.

Let

$$
L(s)=\sum_{n=1}^{\infty} \frac{b_{n}}{n^{s}}
$$

be a Dirichlet series that converges absolutely in a half plane, $\Re(s)>\sigma_{1}$.

Let

$$
\Lambda(s)=Q^{s}\left(\prod_{j=1}^{a} \Gamma\left(\kappa_{j} s+\lambda_{j}\right)\right) L(s),
$$

with $Q, \kappa_{j} \in \mathbb{R}^{+}, \Re\left(\lambda_{j}\right) \geqslant 0$, and assume that:

(1) $\Lambda(s)$ has a meromorphic continuation to all of $\mathbb{C}$ with simple poles at $s_{1}, \ldots, s_{\ell}$ and corresponding residues $r_{1}, \ldots, r_{\ell}$;

(2) $\Lambda(s)=\varepsilon \overline{\Lambda(1-\bar{s})}$ for some $\varepsilon \in \mathbb{C},|\varepsilon|=1$;

(3) for any $\sigma_{2} \leqslant \sigma_{3}, L(\sigma+i t)=O\left(\exp t^{A}\right)$ for some $A>0$, as $|t| \rightarrow \infty, \sigma_{2} \leqslant \sigma \leqslant \sigma_{3}$, with $A$ and the constant in the 'Oh' notation depending on $\sigma_{2}$ and $\sigma_{3}$.

Note that (3.2) expresses the functional equation in more general terms than (2.5), but it is a simple matter to unfold the definition of $\Gamma_{\mathbb{R}}$ and $\Gamma_{\mathbb{C}}$.

To obtain a smoothed approximate functional equation with desirable properties, Rubinstein [15] introduces an auxiliary function. Let $g: \mathbb{C} \rightarrow \mathbb{C}$ be an entire function that, for fixed $s$, satisfies

$$
\left|\Lambda(z+s) g(z+s) z^{-1}\right| \rightarrow 0
$$

as $|\Im z| \rightarrow \infty$, in vertical strips, $-x_{0} \leqslant \Re z \leqslant x_{0}$. The smoothed approximate functional equation has the following form. 
Theorem 3.1. For $s \notin\left\{s_{1}, \ldots, s_{\ell}\right\}$, and $L(s), g(s)$ as above,

$$
\Lambda(s) g(s)=\sum_{k=1}^{\ell} \frac{r_{k} g\left(s_{k}\right)}{s-s_{k}}+Q^{s} \sum_{n=1}^{\infty} \frac{b_{n}}{n^{s}} f_{1}(s, n)+\varepsilon Q^{1-s} \sum_{n=1}^{\infty} \frac{\overline{b_{n}}}{n^{1-s}} f_{2}(1-s, n)
$$

where

$$
\begin{aligned}
f_{1}(s, n) & :=\frac{1}{2 \pi i} \int_{\nu-i \infty}^{\nu+i \infty} \prod_{j=1}^{a} \Gamma\left(\kappa_{j}(z+s)+\lambda_{j}\right) z^{-1} g(s+z)(Q / n)^{z} d z, \\
f_{2}(1-s, n) & :=\frac{1}{2 \pi i} \int_{\nu-i \infty}^{\nu+i \infty} \prod_{j=1}^{a} \Gamma\left(\kappa_{j}(z+1-s)+\overline{\lambda_{j}}\right) z^{-1} g(s-z)(Q / n)^{z} d z
\end{aligned}
$$

with $\nu>\max \left\{0,-\Re\left(\lambda_{1} / \kappa_{1}+s\right), \ldots,-\Re\left(\lambda_{a} / \kappa_{a}+s\right)\right\}$.

In our examples $L(s)$ continues to an entire function, so the first sum in (3.4) does not appear. For fixed $Q, \kappa, \lambda, \varepsilon$, and sequence $b_{n}$, and $g(s)$ as described below, the right side of (3.4) can be evaluated to high precision.

A reasonable choice for the weight function is

$$
g(s)=e^{i b s+c s^{2}}
$$

which by Stirling's formula satisfies (3.3) if $c>0$, or if $c=0$ and $|b|<\pi d / 4$, where $d$ is the degree of the $L$-function. Rubinstein [15] uses such a weight function with $b$ chosen to balance the size of the terms in the approximate functional equation, minimizing the loss in precision in the calculation. In this paper we exploit the fact that there are many choices of weight function, and so there are many ways to evaluate the $L$-function. We combine those calculations to extract as much information as possible from the known Dirichlet coefficients. This idea is described in the next section.

In the computations we carry out below, we find it more convenient to use the Hardy $Z$-function in our computations instead of the $L$-function itself. The function $Z$ associated to an $L$-function $L$ is defined by the properties: $Z\left(\frac{1}{2}+i t\right)$ is a smooth function which is real if $t$ is real, and $\left|Z\left(\frac{1}{2}+i t\right)\right|=\left|L\left(\frac{1}{2}+i t\right)\right|$.

\section{Exploiting the test function in the approximate functional equation}

If we let $g(s)=1$ and $s=\frac{1}{2}+10 i$ in the approximate functional equation (3.4) for the standard (degree 5) $L$-function of $\Upsilon_{20}$, we get

$$
\begin{aligned}
Z\left(\frac{1}{2}+10 i, \Upsilon_{20}, \operatorname{stan}\right)= & -1835.424-395.011 b_{2}+1012.179 b_{3}+1906.603 b_{4} \\
& +2226.503 b_{5}+\ldots+6.840 \times 10^{-9} b_{82} \\
& +5.132 \times 10^{-9} b_{83}+\ldots+3.205 \times 10^{-16} b_{149} \\
& +2.564 \times 10^{-16} b_{150}+\ldots
\end{aligned}
$$

If instead we let $g(s)=e^{-3 i s / 2}$ and keep $s=\frac{1}{2}+10 i$ then we have

$$
\begin{aligned}
Z\left(\frac{1}{2}+10 i, \Upsilon_{20}, \operatorname{stan}\right)= & 1.66549+1.39643 b_{2}-0.658439 b_{3}+0.726149 b_{4} \\
& -0.88227 b_{5}+\ldots+1.532 \times 10^{-8} b_{82} \\
& +1.271 \times 10^{-8} b_{83}+\ldots+3.514 \times 10^{-14} b_{149} \\
& +2.309 \times 10^{-14} b_{150}+\ldots
\end{aligned}
$$


Note that neither of the above expressions appears optimal: the first involves large coefficients, which will lead to a loss of precision. In the second, the terms do not decrease as quickly, so one must use more coefficients to achieve a given accuracy. An observation that we exploit is the fact that the above are just two among a large number of expressions for the value of the $L$-function at $\frac{1}{2}+10 i$.

Recall that by $[\mathbf{1 2}, \mathbf{1 9}]$ we know the Satake parameters of $\Upsilon_{20}$ for all $p \leqslant 79$. Therefore we recognize two types of terms in the approximate functional equation, as illustrated in the above examples. There are terms for which we know the Dirichlet coefficients, such as $b_{5}, b_{82}$, and $b_{150}$. And there are terms with an unknown Dirichlet coefficient, such as $b_{83}$ or $b_{149}$. Actually, there is a third type of term, such as $b_{166}=b_{2} b_{83}$ which is 'partially unknown'. We can estimate the unknown terms by applying the Ramanujan bound to the Dirichlet coefficient, and evaluate everything else precisely. Thus, once we choose a test function, we can evaluate an $L$-function at a given point as

$$
Z(s)=\text { calculated_value }(s) \pm \text { error_estimate }(s),
$$

where both the calculated value and the error estimate are functions of the test function and the set of known Dirichlet coefficients. For later use, we write

$$
\text { error_estimate }(s)=\sum_{n: b_{n} \text { unknown }} \delta_{n}(g, s) b_{n}
$$

where the $\delta_{n}(g, s)$ is the coefficient of $b_{n}$ in (3.4). The product of $\delta_{n}(g, s)$ and the Ramanujan bound for $b_{n}$ is an upper bound for the error contributed to computation by the unknown coefficient $b_{n}$. In the calculations described below, we directly evaluate the contributions from the first 2000 Dirichlet coefficients. For $n \leqslant 2000$ we use the calculated value of $\delta_{n}(g, s)$ and the Ramanujan bound for $b_{n}$ to estimate the contribution. This is the main source of the error term in (4.4).

While there are rigorous bounds for the contribution of the tail to the error (see, for example, [13, Propositions 3.7 and 3.9]) we do not make use of them for two reasons. First, those general bounds are much larger than what is actually observed in our examples. For instance, in (4.2) it appears that by the 150 th term the contribution is less than $10^{-13}$, and this is confirmed by further computation (to thousands of terms), showing a steady decrease at the expected rate. But the general bounds of [13] require about 8000 terms before the predicted contribution drops below $10^{-13}$. Second, we consider our method to be experimental and, as such, did not emphasize being so careful with the bounds and instead, relied on observation and intuition. We think, as illustrated in examples below, the fact that we were able to obtain consistent values for our calculations of $L$-functions is evidence that the results are correct and in principle could be made rigorous.

Using the known $b_{n}$ and applying the Ramanujan bound (2.6) to (4.1) we get

$$
Z\left(\frac{1}{2}+10 i, \Upsilon_{20}, \operatorname{stan}\right)=3.0393070838 \pm 3.12 \times 10^{-8} .
$$

And for (4.2) we get

$$
Z\left(\frac{1}{2}+10 i, \Upsilon_{20}, \operatorname{stan}\right)=3.0393070808 \pm 7.10 \times 10^{-8} .
$$

In Figure 1 we show the calculated value and error estimate for $Z\left(\frac{1}{2}+10 i, \Upsilon_{20}\right.$, stan $)$ when evaluated with test functions of the form $g(s)=e^{-i \beta s}$. Note that the vertical axis in the figure is on a $\log$ scale.

As Figure 1 shows, there is a wide range of $\beta$ for which it is possible to determine $\Lambda\left(\frac{1}{2}+10 i, \Upsilon_{20}\right.$, stan $)$ with some accuracy. With the optimal choice of $\beta$ the error estimate is approximately $4 \times 10^{-10}$. 


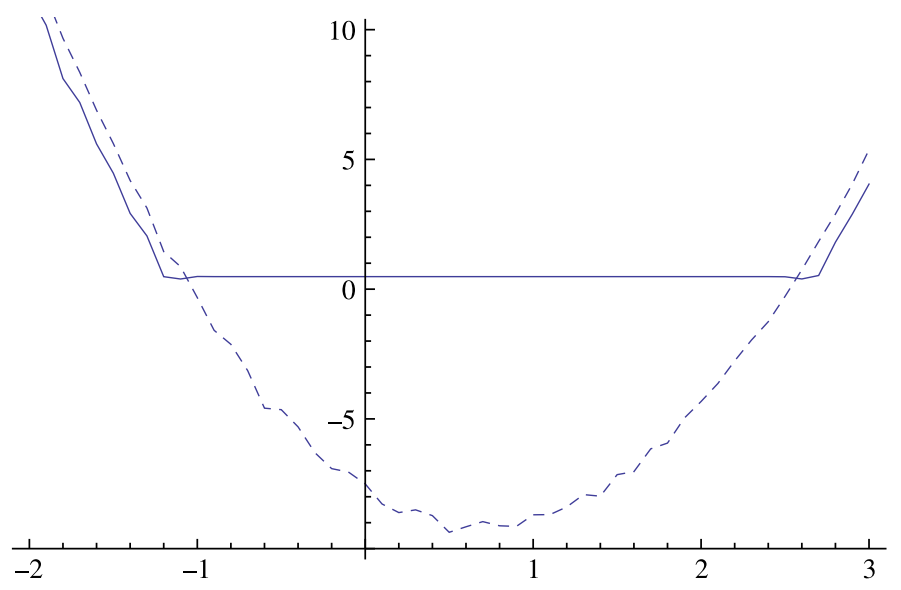

FiguRE 1. The solid line is the calculated value and the dashed line is the error estimate in computing $Z\left(\frac{1}{2}+10 i, \Upsilon_{20}\right.$, stan $)$ using the available Dirichlet coefficients with the weight function $g(s)=e^{-i \beta s}$ where $\beta$ is given along the horizontal axis. The vertical axis is $\log _{10}$ of (the absolute value of) the actual value. For quite a wide range of test functions, the value of the $Z$-function at $\frac{1}{2}+10 i$ is determined with some accuracy, achieving around 10 decimal digits of accuracy with the optimal choice of $\beta$.

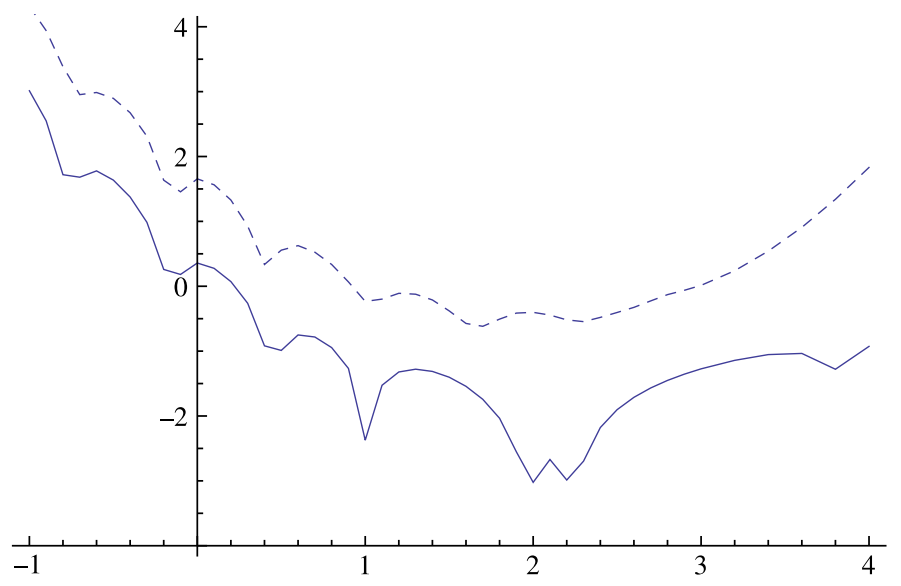

Figure 2. The analogue of Figure 1 for $Z\left(\frac{1}{2}+5 i, \Upsilon_{20}\right.$, adj) with the weight function $g(s)=e^{-i \beta s+(1 / 500)(s-5 i)^{2}}$ where $\beta$ is given along the horizontal axis.

Figure 2 shows the calculated value and error estimate for the adjoint (degree 10) $L$-function $Z\left(\frac{1}{2}+5 i, \Upsilon_{20}\right.$, adj) when evaluated with test functions of the form $g(s)=e^{-i \beta s+(1 / 500)(s-5 i)^{2}}$.

As Figure 2 shows, every test function of the given form leads to an error which is larger than the calculated value. Thus, we can determine that $\mid Z\left(\frac{1}{2}+5 i, \Upsilon_{20}\right.$, adj $) \mid<0.25$, but with individual test functions of the given form we cannot even determine if the actual value is positive or negative.

We now introduce a new idea for increasing the accuracy of these calculations.

\subsection{Optimizing the test function}

In Figure 1 we see that there are many values of the parameters in the test function which give reasonable results. If there is some degree of independence in the errors, then there is hope for obtaining a smaller error by combining the results of those separate calculations. 
Write $Z\left(s, \Upsilon_{20}\right.$, stan, $\left.\beta\right)$ for the output of the approximate functional equation with weight function $g(s)=e^{-i \beta s}$. Consider

$$
Z\left(s, \Upsilon_{20}, \operatorname{stan}\right)=\sum_{j=1}^{J} c_{\beta_{j}} Z\left(s, \Upsilon_{20}, \operatorname{stan}, \beta_{j}\right)
$$

where $\sum c_{\beta_{j}}=1$. We make the specific choices

$$
\begin{aligned}
s & =\frac{1}{2}+10 i \\
\left(\beta_{1}, \beta_{2}, \beta_{3}, \beta_{4}, \beta_{5}\right) & =\left(\frac{1}{10}, \frac{2}{10}, \frac{3}{10}, \frac{4}{10}, \frac{5}{10}\right), \\
\left(c_{\beta_{1}}, c_{\beta_{2}}, c_{\beta_{3}}, c_{\beta_{4}}, c_{\beta_{5}}\right) & =(0.03150,0.18061,0.36563,0.31421,0.10801) .
\end{aligned}
$$

Recall that all decimal numbers are truncations of the actual values; one requires much higher precision than the displayed numbers in order to obtain the answers below. With the choices in (4.8), after substituting the known Dirichlet coefficients and then using the Ramanujan bound, we find

$$
\begin{aligned}
Z\left(s, \Upsilon_{20}, \operatorname{stan}\right)= & 3.03930708648952782778+2.688 \cdot 10^{-19} b_{83}+\ldots-1.147 \cdot 10^{-16} b_{107} \\
& +\ldots-5.291 \cdot 10^{-18} b_{137}+\ldots+1.216 \cdot 10^{-23} b_{199}+\ldots \\
= & 3.039307086489527827 \pm 4.73 \cdot 10^{-15} .
\end{aligned}
$$

Thus, by averaging only five evaluations of the $L$-function, the error decreased by a factor of almost $10^{-5}$.

The weights $c_{\beta_{j}}$ in (4.8) were determined by finding the least-squares fit to

$$
\sum_{n: b_{n} \text { unknown }} \operatorname{Ram}\left(b_{n}\right)^{2}\left(\sum_{j} c_{\beta_{j}} \delta_{n}\left(\beta_{j}, \frac{1}{2}+10 i\right)\right)^{2}=0,
$$

where $\operatorname{Ram}\left(b_{n}\right)$ is the Ramanujan bound (2.6) for $b_{n}$, subject to $\sum c_{\beta_{j}}=1$. Note that in our actual examples the vast majority of unknown coefficients have prime index, so the $\operatorname{Ram}\left(b_{n}\right)$ weighting is not important, but we include it for completeness. For the calculations in this paper, we use the $n<1000$ for which $b_{n}$ is unknown in (4.10).

The error estimate in (4.9) is an $L^{1}$ estimate, not the $L^{2}$ estimate as shown in (4.10), so actually it is possible to choose slightly better weights than those used in our example. In $\S 5$ we show how to obtain the optimal result that can arise from combining different evaluations of the $L$-function, but for now we merely wish to illustrate the seemingly surprising fact that appropriately combining several evaluations can vastly decrease the error.

\subsection{Results}

In Figure 3 we plot the error obtained by combining varying numbers of weight functions, where we evaluate $Z\left(\frac{1}{2}+10 i, \Upsilon_{20}\right.$, stan $)$ with a weight function $g(s)=e^{i \beta s}$ with $\beta=j / 10$ with $-10 \leqslant j \leqslant 25$. The horizontal axis is the number of terms averaged, where we start with $\beta=\frac{1}{2}$ and first use those $\beta$ which are closest to $\frac{1}{2}$. The vertical axis is the error estimate on a $\log _{10}$ scale. The lowest point on the graph, when we average all 36 evaluations, corresponds to

$$
\begin{aligned}
& Z\left(\frac{1}{2}+10 i, \Upsilon_{20}, \operatorname{stan}\right) \\
& \quad=3.0393070864895284810824603284422250910 \pm 2.79 \times 10^{-35} .
\end{aligned}
$$

It is not clear from Figure 3 whether one would expect to obtain an arbitrarily small error by combining sufficiently many test functions in the approximate functional equations. This is discussed in $\S 6$. 


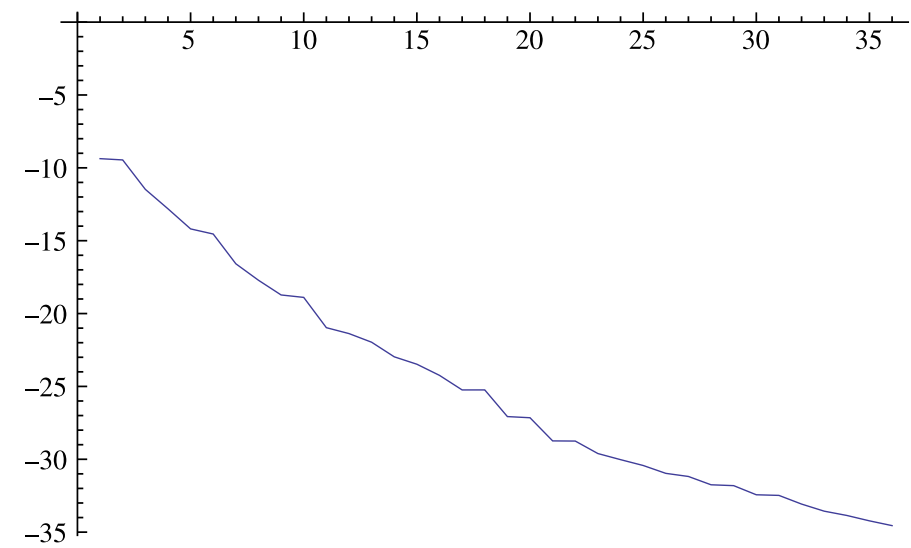

FiguRE 3. The error obtained from a least-squares minimization of the error for combining $n$ evaluations of $Z\left(\frac{1}{2}+10 i, \Upsilon_{20}\right.$, stan) using the weight functions $g(s)=e^{-i \beta s}$ with $\beta=j / 10$. The horizontal axis is $n$ and the vertical axis is $\log _{10}$ of the resulting error estimate.

We briefly describe the corresponding calculations for $Z\left(\frac{1}{2}+5 i, \Upsilon_{20}\right.$, adj). Recall that, as shown in Figure 2, with a single test function of the standard form we are not able to determine whether that value is positive or negative. Now we combine five evaluations in the analogous way,

$$
Z\left(s, \Upsilon_{20}, \operatorname{adj}\right)=\sum_{j=1}^{J} c_{\beta_{j}} Z\left(s, \Upsilon_{20}, \operatorname{adj}, \beta_{j}\right)
$$

where $\sum c_{\beta_{j}}=1$. Here the weight function is $g(s)=e^{-i \beta s+(s-5 i)^{2} / 500}$.

We make the specific choices

$$
\begin{aligned}
s & =\frac{1}{2}+5 i \\
\left(\beta_{1}, \beta_{2}, \beta_{3}, \beta_{4}, \beta_{5}\right) & =\left(\frac{3}{5}, \frac{6}{5}, \frac{9}{5}, \frac{12}{5}, \frac{15}{5}\right) \\
\left(c_{\beta_{1}}, c_{\beta_{2}}, c_{\beta_{3}}, c_{\beta_{4}}, c_{\beta_{5}}\right) & =(0.035863,0.33504,0.47934,0.13827,0.01146) .
\end{aligned}
$$

The result is

$$
Z\left(\frac{1}{2}+5 i, \Upsilon_{20}, \operatorname{adj}\right)=0.01556 \pm 0.0049
$$

So we have determined that $Z\left(\frac{1}{2}+5 i, \Upsilon_{20}\right.$, adj $)$ is positive, but we can only be certain of one significant figure in its decimal expansion. Using this method, the best result we were able to obtain, by averaging eleven evaluations, is

$$
Z\left(\frac{1}{2}+5 i, \Upsilon_{20}, \operatorname{adj}\right)=0.01558768 \pm 0.00016
$$

Averaging more weight function actually makes the result worse. The explanation is simple: since the adjoint $L$-function has high degree, the error terms $\delta_{n}$ decrease very slowly. The least-squares fit does not properly take into account the contribution of a large number of small terms, so the least-squares fit actually has a large $L^{1}$ norm.

In the next section we describe some 'sanity checks' on our method, as suggested by the referee. Then, in $\S 5$, we introduce a different method which avoids some of the shortcomings in the least-squares method.

\subsection{The method applied to known examples}

We check that our method gives correct results in some cases where it is possible to evaluate the $L$-function using another method. 
First we consider $L(s, \Delta)$, where $\Delta$ is the (unique) weight 12 cusp form for $\operatorname{SL}(2, \mathbb{Z})$, which satisfies the functional equation

$$
\Lambda(s, \Delta):=\Gamma_{\mathbb{C}}\left(s+\frac{11}{2}\right) L(s, \Delta)=\Lambda(1-s, \Delta) .
$$

We will evaluate $Z\left(\frac{1}{2}+100 i, \Delta\right)$ without using any Dirichlet coefficients, other than the leading coefficient 1 . That is, all we know about the $L$-function is its functional equation and the fact that its Dirichlet coefficients satisfy the Ramanujan bound.

In the approximate functional equation we will use the test function $g_{\beta}(s)=$ $e^{-i \beta s+(s-100 i)^{2} / 100}$, for $\beta=-\frac{30}{20},-\frac{29}{20}, \ldots, \frac{69}{20}, \frac{70}{20}$. That is a total of 101 evaluations. Using the least-squares method described previously, we find 101 coefficients $c_{\beta}$ with $\sum c_{\beta}=1$. Forming the weighted sum of the 101 evaluations of $Z\left(\frac{1}{2}+100 i, \Delta\right)$ and estimating the unknown terms as described previously, we find

$$
\begin{aligned}
& Z\left(\frac{1}{2}+100 i, \Delta\right) \\
& \quad=-0.233906591556845205706582417137279238114100783 \pm 3.28 \times 10^{-42} .
\end{aligned}
$$

The given digits are correct to the claimed accuracy: the last three digits shown should be 880 , and the actual difference between the calculated value and the true value is $9.66 \times 10^{-44}$.

Next we consider the $L$-function associated to a weight 24 cusp form for $\operatorname{SL}(2, \mathbb{Z})$. Note that $S_{24}(\mathrm{SL}(2, \mathbb{Z}))$ is two dimensional, and every cusp form $f$ in that vector space satisfies the functional equation

$$
\Lambda(s, f):=\Gamma_{\mathbb{C}}\left(s+\frac{23}{2}\right) L(s, f)=\Lambda(1-s, f) .
$$

We will attempt to evaluate $Z\left(\frac{1}{2}+100 i, f\right)$ using as few coefficients as possible. Because there is more than one function satisfying (4.18), it seems obvious that we cannot evaluate such an $L$-function without knowing any coefficients.

If we assume the cusp form $f$ is a Hecke eigenform, then the Dirichlet coefficient $b_{2}$ determines the coefficients $b_{4}, b_{8}$, etc, and it also allows us to eliminate every even-index Dirichlet coefficient as an 'unknown'. Since that does not seem like an adequately strenuous test of the method, instead we will assume nothing about the Dirichlet series except the functional equation (4.18) and a bound on the Dirichlet coefficients. We will assume a Ramanujan bound of the form $\left|b_{n}\right| \leqslant C_{f} d(n)$, where $d(n)$ is the divisor function and $C_{f}$ is a constant depending only on the cusp form $f$. If $f$ is a Hecke eigenform then $C_{f}=1$, and if $f=A f_{1}+B f_{2}$ where $f_{1}, f_{2} \in S_{24}(\mathrm{SL}(2, \mathbb{Z}))$ are the Hecke eigenforms, then $C_{f}=|A|+|B|$.

Using the same 101 test functions as in the case of $L(s, \Delta)$, and choosing 101 weights to minimize the contribution of $b_{3}, b_{4}, \ldots$, we find

$$
\begin{aligned}
Z\left(\frac{1}{2}+100 i, f\right)= & 1.870426534029268899143339193910896103506087410 b_{1} \\
& +1.125008886302338484473484421487863753620660254 b_{2} \\
& \pm C_{f} \times 2.86 \times 10^{-43} .
\end{aligned}
$$

Thus, we can evaluate $Z\left(\frac{1}{2}+100 i, f\right)$ to 42 decimal places, knowing (up to a normalizing constant) only one Dirichlet coefficient. The values of $b_{2}$ for the two Hecke eigenforms, in the analytic normalization, are

$$
b_{2}=\frac{540 \pm 12 \sqrt{144169}}{2^{32 / 2}},
$$

and inserting those values finds that (4.19) is correct.

Our third check on the method is to evaluate a degree $10 \mathrm{~L}$-function which can be also evaluated in an independent way. To give a reasonable match with the case of $L\left(\frac{1}{2}+5 i, \Upsilon_{20}\right.$, adj $)$, we consider $L\left(\frac{1}{2}+5 i, f\right)^{5}$, where $f$ is a weight 24 cusp form for $\operatorname{SL}(2, \mathbb{Z})$. In other words, 
the same $L$-function as in the previous example, except that we take its 5 th power and evaluate at $\frac{1}{2}+5 i$. Note that this time there are two $L$-functions with the given functional equation, with known values

$$
\begin{aligned}
L\left(\frac{1}{2}+5 i, f_{1}\right)^{5} & =(-3.0527819)^{5}=-265.14223, \\
L\left(\frac{1}{2}+5 i, f_{2}\right)^{5} & =(-0.7404879)^{5}=-0.2226331 .
\end{aligned}
$$

We will assume that we know the Euler factors up through $p=79$, just as in the case of $L\left(\frac{1}{2}+5 i, \Upsilon_{20}\right.$, adj). If we only use a single test function of the standard form, then the best error we can obtain is comparable to what we found in the previous degree 10 case

$$
\begin{aligned}
& L\left(\frac{1}{2}+5 i, f_{1}\right)^{5}=-265.204 \pm 0.314 \\
& L\left(\frac{1}{2}+5 i, f_{2}\right)^{5}=-0.22193 \pm 0.233
\end{aligned}
$$

Using the test functions $g_{\beta}(s)=e^{-i \beta s+(s-5 i)^{2} / 500}$, selecting the seven values of $\beta$ in the set $\left\{-\frac{10}{10},-\frac{2}{10}, \frac{4}{10}, \frac{6}{10}, \frac{11}{10}, \frac{17}{10}, \frac{18}{10}, \frac{25}{10}\right\}$, and using the least-squares method to find suitable weights, we find

$$
\begin{aligned}
& L\left(\frac{1}{2}+5 i, f_{1}\right)^{5}=-265.14224 \pm 0.00117 \\
& L\left(\frac{1}{2}+5 i, f_{2}\right)^{5}=-0.222664 \pm 0.00186 .
\end{aligned}
$$

Averaging only seven evaluations decreased the error by a factor of more than 100 , and we see that the calculated values are in fact correct. This confirms that our method gives consistent results in cases of comparably complicated $L$-functions for which it is possible to give an independent check on the calculations.

\section{Linear programming}

In this section we view the evaluation of the $L$-function as an optimization problem. For example, we can view the equality of the expressions in (4.1) and (4.2) as a constraint on the value of the $L$-function. Thus, the same calculations which were used as input for the least-squares method described in the previous section can also be used as input to a linear programming problem.

We set up the linear programming problem in the following way. Let $Z\left(\frac{1}{2}+10 i, \Upsilon_{20}, \operatorname{stan}, g_{j}\right)$ denote the evaluation of $Z\left(\frac{1}{2}+10 i, \Upsilon_{20}\right.$, stan $)$ using the weight function $g_{j}$ in the approximate functional equation. One evaluation, $Z\left(\frac{1}{2}+10 i, \Upsilon_{20}\right.$, stan, $\left.g_{1}\right)$, is taken as the objective. The other evaluations are taken in pairs and

$$
Z\left(\frac{1}{2}+10 i, \Upsilon_{20}, \operatorname{stan}, g_{1}\right)-Z\left(\frac{1}{2}+10 i, \Upsilon_{20}, \text { stan, } g_{j}\right)=0
$$

is interpreted as a constraint. The other constraints come from the Ramanujan bound on the unknown coefficients.

In the above description there are infinitely many unknowns and constraints. We eliminate the unknown coefficients $b_{n}$ with $n>1000$ by using the Ramanujan bound, replacing the equality (5.1) by a pair of inequalities. Thus, we have a straightforward linear programming problem, which we use to determine the minimum and maximum possible values of $Z\left(\frac{1}{2}+10 i, \Upsilon_{20}\right.$, stan $)$.

We implemented this idea using the same set of test functions we used for the leastsquares method. The calculations were done in Mathematica [20], using the built-in LinearProgramming function with the Method $\rightarrow$ Simplex option. In all cases the linear programming method gave better results, but not spectacularly better. Figure 4 shows the ratio of the errors in the results of the two methods, on a $\log _{10}$ scale. For example, when using 30 equations the error from the linear programming approach was approximately $1 / 10$ the error from the least-squares method. 


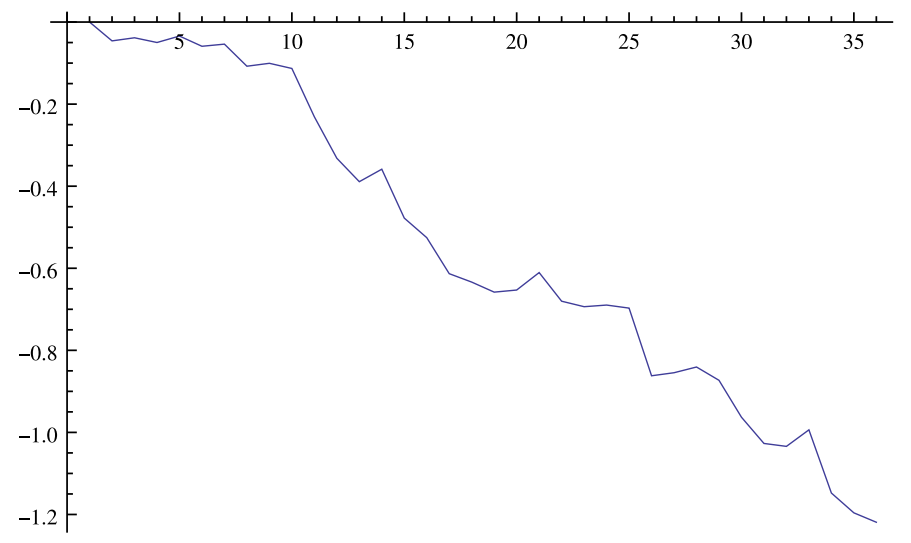

FiguRE 4 . The ratio of the errors in the linear programming and least-squares methods for evaluating $Z\left(\frac{1}{2}+10 i, \Upsilon_{20}\right.$, stan $)$ using $n$ equations. The horizontal axis is $n$ and the vertical axis is $\log _{10}$ of the ratio of errors.

\subsection{Computing unknown coefficients}

An interesting side-effect of the linear programming approach is that it allows us to obtain information about the unknown coefficients. Instead of treating the value of the $L$-function as the objective, we can use an unknown coefficient as the objective. Note that all the other constraints in the problem are unchanged. Using the same method as described above we find the following coefficients of the standard $L$-function of $\Upsilon_{20}$

$$
\begin{aligned}
& b_{83}=0.4884558312724 \pm 2.4 \times 10^{-12}, \\
& b_{89}=0.10561760640 \pm 2.7 \times 10^{-10} \\
& b_{97}=0.468135808 \pm 1.5 \times 10^{-7} .
\end{aligned}
$$

Since the eigenvalues of $\Upsilon_{20}$ are integral and of a known size, if we were to know $b_{83}$ to 35 digits, we would determine it exactly. This is computationally expensive but perhaps not as expensive as if we were to compute more Fourier coefficients of $\Upsilon_{20}$.

These results can be checked once more Fourier coefficients of $\Upsilon_{20}$ are computed. By the $n^{4}$ argument given in the introduction, computing exact values of $b_{p}$ for $p \leqslant 97$ will take about twice as much work as it took to compute those for $p \leqslant 79$.

\section{Conclusions and further questions}

We have shown that, at the cost of a lot of computation, one can evaluate an $L$-function to high precision using only a small number of coefficients. That this is theoretically possible is not surprising: $L$-functions are very special objects, and the data we have for the $L$-function considered here (the functional equation and the first several coefficients) presumably specify the $L$-function uniquely. Thus, in an abstract sense there is no new information in the missing coefficients. But the question remains as to whether our methods accomplish this in practice.

Question 6.1. Can the method of calculating an $L$-function by evaluating the approximate functional equation and then averaging to minimize the contributions of the unknown coefficients, determine numerical values of the $L$-function to arbitrary accuracy? 
Because the approximate functional equation requires a huge number of terms to evaluate a high-degree $L$-function, it would be significant if the weights we obtained by our methods could be determined without actually calculating all the terms with unknown coefficients.

Problem 6.2. Devise a method of determining an optimal weight function in the approximate functional equation without first calculating a large number of terms which do not actually contribute to the final answer.

Implicit in the above problem is the requirement that one knows the calculated value as well as an estimate of the error.

Problem 6.3. Is there any meaning to the weights determined by the least-squares method?

The weights which appear in our least-squares method depend on the point at which the $L$-function is evaluated. It might be helpful to consider a case where there are two $L$-functions with the same functional equation, such as the spin $L$-functions of $\operatorname{Sp}(4, \mathbb{Z})$ Siegel modular forms of weight $k \geqslant 22$.

Without progress on these problems, or a completely new method, there is little hope of making extensive computations of high-degree $L$-functions.

Acknowledgements. We thank the referee for suggesting we include the material in $\S 4.3$.

\section{References}

1. A. N. Andrianov, 'Euler products that correspond to Siegel's modular forms of genus 2', Uspehi Mat. Nauk 29 (1974) 43-110.

2. A. N. Andrianov, Quadratic forms and Hecke operators, Grundlehren der Mathematischen Wissenschaften [Fundamental Principles of Mathematical Sciences] 286 (Springer, Berlin, 1987).

3. S. BLOCH and K. KATO, ' $L$-functions and Tamagawa numbers of motives', The Grothendieck Festschrift, Vol. I, Progress in Mathematics 86 (Birkhäuser Boston, Boston, MA, 1990) 333-400.

4. S. BöchERER, 'Über die Funktionalgleichung automorpher $L$-Funktionen zur Siegelschen Modulgruppe', J. reine angew. Math. 362 (1985) 146-168.

5. S. Böcherer, 'Bemerkungen über die Dirichletreihen von Koecher und Maaß (Remarks on the Dirichlet series of Koecher and Maßß)', Technical Report, Math. Gottingensis, Schriftenr. Sonderforschungsbereichs Geom. Anal. 68, 36 S., 1986.

6. J. Brian Conrey, D. W. Farmer, J. P. Keating, M. O. Rubinstein and N. C. Snaith, 'Integral moments of $L$-functions', PLMS 91 (2005) 33-104.

7. J. Brian Conrey, D. W. Farmer and M. R. Zirnbauer, 'Autocorrelation of ratios of $L$-functions', Commun. Number Theory Phys. 2 (2008) 593-636.

8. T. Dokchitser, 'Computing special values of motivic L-functions', Exp. Math. 13 (2004) 137-149.

9. D. W. FARmer, N. C. Ryan and R. Schmidt, 'Testing the functional equation of a high-degree Euler product', Pacific J. Math. 253 (2011) 349-366.

10. J. P. Keating and N. C. Snaith, 'Random matrices and L-functions', J. Phys. A 36 (2003) 2859-2881; Random matrix theory.

11. H. KLINGEn, Introductory lectures on Siegel modular forms, Cambridge Studies in Advanced Mathematics 20 (Cambridge University Press, Cambridge, 1990).

12. W. Kohnen and M. Kuss, 'Some numerical computations concerning spinor zeta functions in genus 2 at the central point', Math. Comp. 71 (2002) 1597-1607.

13. P. Molin, 'Intégration numérique et calculs de fonctions L', PhD Thesis, Institut de Mathématiques de Bordeaux, 2010.

14. A. Pitale, A. Saha and R. Schmidt, 'Transfer of Siegel cusp forms of degree 2', Mem. Amer. Math. Soc., to appear, arXiv:1106.5611.

15. M. O. Rubinstein, 'Computational methods and experiments in analytic number theory', Recent perspectives in random matrix theory and number theory, London Mathematical Society Lecture Note Series 322 (Cambridge University Press, Cambridge, 2005) 425-506.

16. N. C. RYAn, 'Computing the Satake $p$-parameters of Siegel modular forms', Preprint, 2004, arXiv:math/0411393. 
17. N. C. Ryan and G. Tornaría, 'A Böcherer-type conjecture for paramodular forms', Int. J. Number Theory 7 (2011) 1395-1411.

18. N.-P. Skoruppa, 'Computations of Siegel modular forms of genus two', Math. Comp. 58 (1992) 381-398.

19. N.-P. Skoruppa, 'Data for rational Siegel eigenforms', Personal website, 2010.

20. Wolfram Research Inc. Mathematica (Version 8.0), 2010.

David W. Farmer

American Institute of Mathematics

360 Portage Ave

Palo Alto, CA 94306

USA

farmer@aimath.org

\author{
Nathan C. Ryan \\ Department of Mathematics \\ Bucknell University \\ Lewisburg, PA 17837 \\ USA \\ nathan.ryan@bucknell.edu
}

